


\title{
Centimeter
}

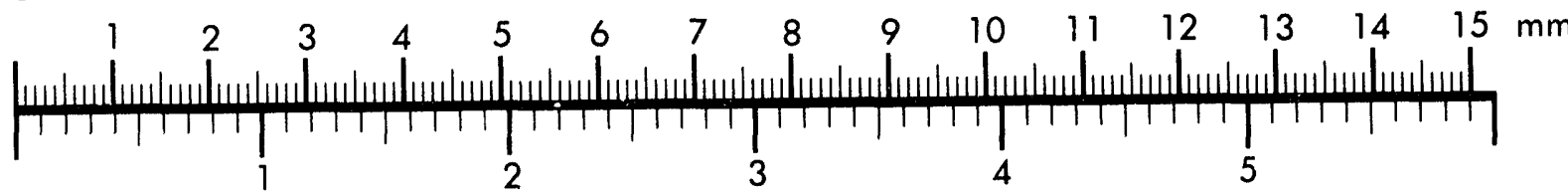
Inches
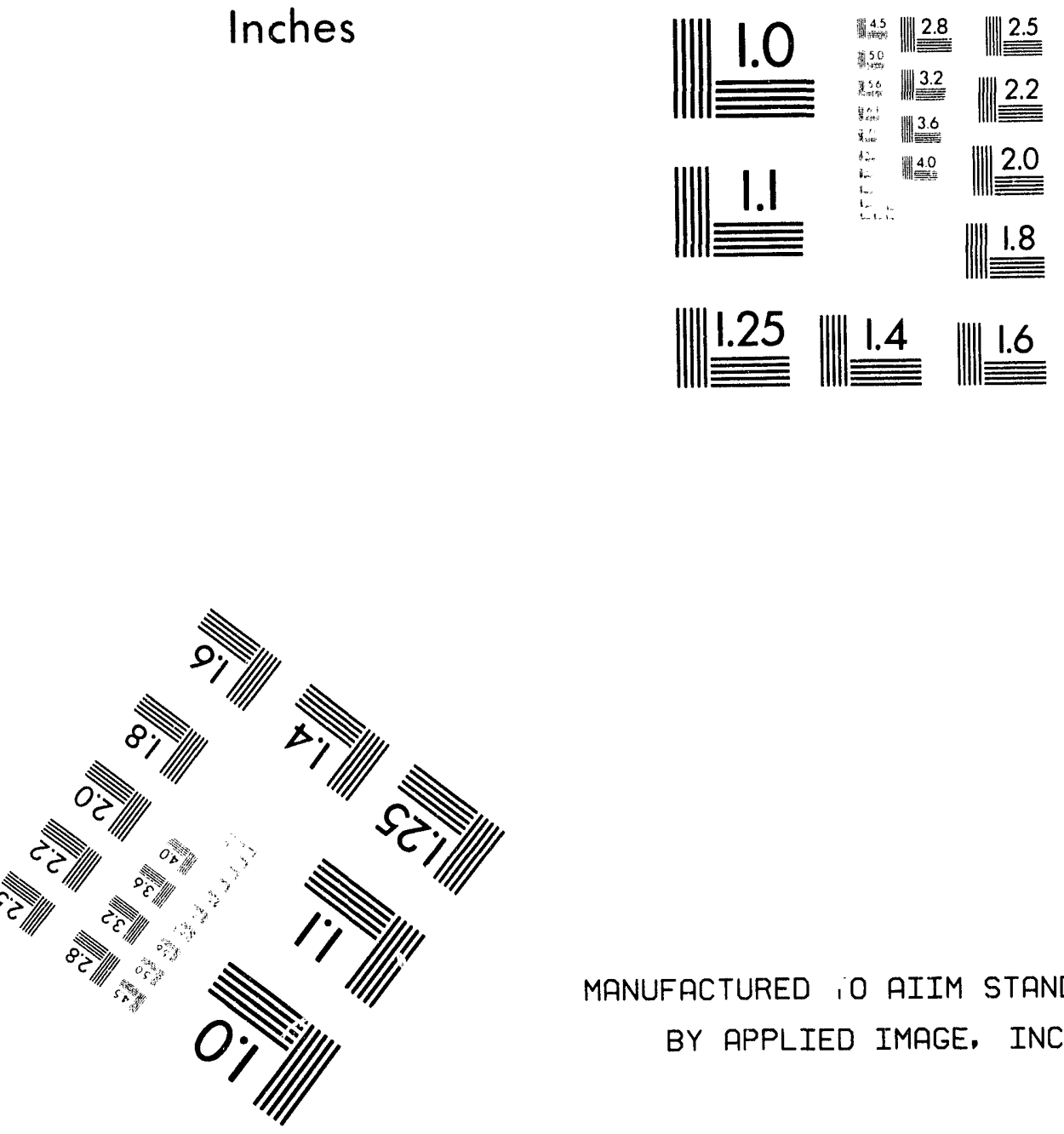

MANUFACTURED iO AIIM STANDARDS

BY APPLIED IMAGE, INC.

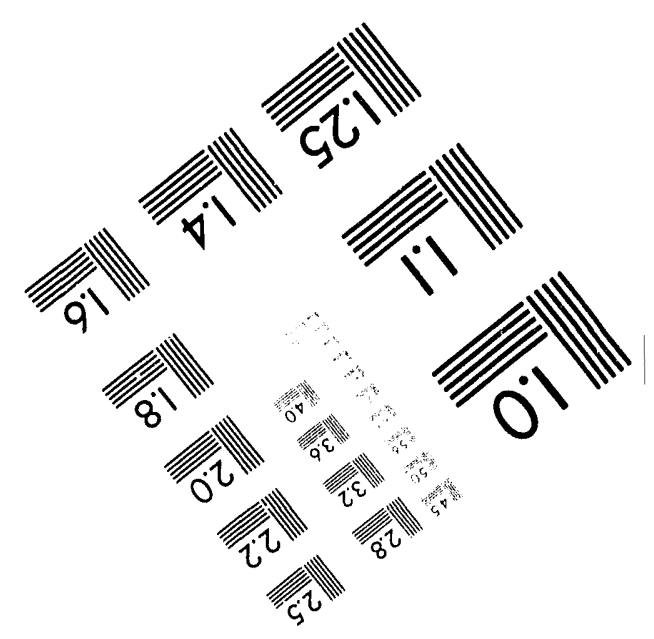



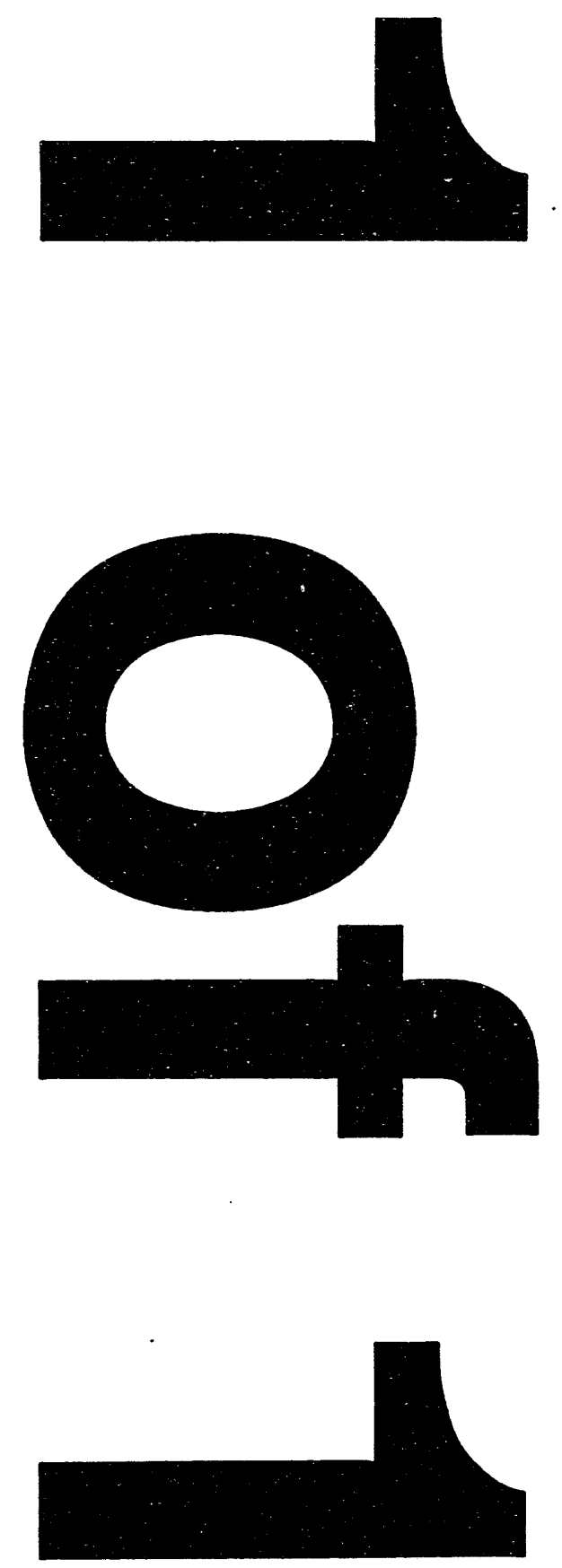
Engineering Physics and Mathematics Division

\section{A METHOD FOR OBTAINING A LEAST SQUARES FIT OF A HYPERPLANE TO UNCERTAIN DATA}

David B. Reister and Max D. Morris

DATE PUBLISHED — May 1994

Research sponsored by the Engineering Research Program

Office of Basic Energy Sciences

U.S. Department of Energy and by the

Naval Air Warfare Center Aircraft Division/API Group

Technology and Development Office

Interagency Agreement No. 2072-E123-A1

U.S. Department of Energy

Prepared by the

OAK RIDGE NATIONAL LABORATORY

Oak Ridge, Tennessee 37831

managed by

MARTIN MARIETTA ENERGY SYSTEMS, INC.

for the

U.S. DEPARTMENT OF ENERGY

under contract DE-AC05-84OR21400 


\section{CONTENTS}





2. ESTIMATION OF A BEST HYPERPLANE ….................................. 3

3. NUMERICAL EXPERIMENTS …................................................ 7

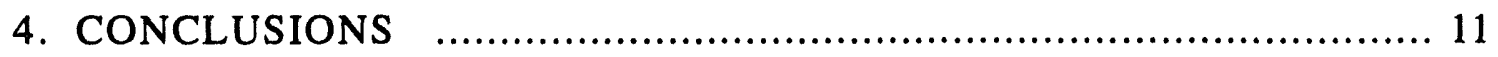






\begin{abstract}
For many least squares problems, the uncertainty is in one of the variables [for example, $y=f(x)$ or $z=f(x, y)]$. However, for some problems, the uncertainty is in the geometric transformation from measured data to Cartesian coordinates and all of the calculated variables are uncertain.

When we seek the best least squares fit of a hyperplane to the data, we obtain an over determined system (we have $n+1$ equations to determine $n$ unknowns). By neglecting one of the equations at a time, we can obtain $n+1$ different solutions for the unknown parameters. However, we cannot average the $n+1$ hyperplanes to obtain a single best estimate. To obtain a solution without neglecting any of the equations, we solve an eigenvalue problem and use the eigenvector associated with the smallest eigenvalue to determine the unknown parameters. We have performed numerical experiments that compare our eigenvalue method to the approach of neglecting one equation at a time.
\end{abstract}




\section{INTRODUCTION}

We became interested in this problem because we wanted to calibrate our laser range cameras. Laser range cameras are powerful tools for defining the Cartesian coordinates of surfaces of objects in an environment. The camera scans a laser beam over a scene and determines the distance (r) by the time required for the beam to reach an object and return. The beam is directed back and forth $(\phi)$ and up and down $(\theta)$ by two rotating mirrors. Thus, the data measured by the camera has the form $(r, \phi, \theta)$. The geometry of the camera can be used to map the measured data to Cartesian coordinates $(\mathbf{x}, \mathbf{y}, \mathbf{z})$.

Since the geometry of the camera may not be known precisely, the cameras can be calibrated by comparing the calculated surface shapes to the known surface shapes. The most simple surface is a plane and many physical objects have planar surfaces. Thus, an important problem in the calibration of range cameras is to find the best (least squares) fit of a plane to a set of $3 \mathrm{D}$ points.

For many least squares problems, the uncertainty is in one of the variables [for example, $y=f(x)$ or $z=f(x, y)]$. However, for this problem, the uncertainty is in the geumetric transformation from measured data to Cartesian coordinates and all three of the calculated variables are uncertain.

When we seek the best least squares fit of a plane to the data, we obtain an over determined system (we have four equations to determine three unknowns). By neglecting one of the equations at a time, we can obtain four different solutions for the unknown parameters. However, we cannot average the 4 planes to obtain a single best estimate. To obtain a solution without neglecting any of the equations, we solve an eigenvalue problem and use the eigenvector associated with the smallest eigenvalue to determine the unknown parameters. We have performed numerical experiments that compare our eigenvalue method to the approach of neglecting one equation at a time. 


\section{ESTIMATION OF A BEST HYPERPLANE}

We will generalize our problem from three dimensions to $\mathrm{n}$ dimensions. We assume that we receive estimates of points ( $p$ ) in an $n$ dimensional space:

$$
p=\left(x_{1}, x_{2}, \ldots, x_{n}\right)
$$

The data will be a sct of points $\left(\mathrm{p}_{\mathrm{j}}\right)$, where $\mathrm{j}$ ranges from 1 to $\mathrm{m}$ :

$$
\mathrm{p}_{\mathrm{j}}=\left(\mathrm{x}_{1 \mathrm{j}}, \mathrm{x}_{2 \mathrm{j}}, \ldots, \mathrm{x}_{\mathrm{nj}}\right)
$$

A hyperplane is defined by the following equation:

$$
d+\sum_{i=1}^{n} a_{i} x_{i}=0
$$

If the parameter vector $a$ is a unit vector, $d$ is the perpendicular distance from the origin to the plane. Although Eq. (3) has $n+1$ parameters (the $n$ vector a and the scalar $d$ ), only $n$ of the parameters are free when a is a unit vector. If we divide Eq. (3) by the distance (d), Eq. (3) depends on the $n$ parameters $\left(a_{i} / d\right)$. We will solve for the parameters $\left(a_{i} / d\right)$ and use the normalization condition to determine $d$ and the vector $a$.

Since the data will not all lie on the hyperplane, we can define an error $\left(e_{j}\right)$ for each point:

$$
e_{j}=d+\sum_{i=1}^{n} a_{i} x_{i j}
$$

We will choose the parameters to minimize the weighted sum of the squares of the errors (L):

$$
L=\sum_{j=1}^{m} w_{j}\left(e_{j}\right)^{2}
$$

where the weights $\left(w_{j}\right)$ are nonnegative and sum to 1.0 . 
Using Eq. (4), L may be written:

$$
L=d^{2}+2 d \sum_{i=1}^{n} a_{i} B_{i}+\sum_{i=1}^{n} \sum_{k=1}^{n} a_{i} a_{k} A_{i k}
$$

where:

$$
\begin{aligned}
A_{i k} & =\sum_{j=1}^{m} w_{j} x_{i j} x_{k j} \\
B_{i} & =\sum_{j=1}^{m} w_{j} x_{i j}
\end{aligned}
$$

The A matrix is symmetric $\left(A_{i k}=A_{k i}\right)$.

For the parameters that minimize $L$, the partial derivative of $L$ with respect to each parameter is zero:

$$
\begin{gathered}
d+\sum_{i=1}^{n} a_{i} B_{i}=0 \\
d B_{k}+\sum_{i=1}^{n} a_{i} A_{i k}=0, \text { for } k=1 \text { to } n
\end{gathered}
$$

Thus, we have $n+1$ equations to determine the $n$ parameters $\left(a_{i} / d\right)$.

Define the real symmetric matrix (D) by:

$$
D=\left[\begin{array}{ll}
1 & B \\
B^{T} & A
\end{array}\right]
$$

where the components of the row vector B are defined by Eq. (8) and the components of the matrix A are defined by Eq. (7). Using D, Eqs. (9) and (10) may be written:

$$
\text { Dy }=0
$$


where the column vector $y$ is defined by:

$$
y=\left[\frac{d}{a}\right]
$$

and the components of the column vector a are the $n$ parameters $\left(a_{i}\right)$.

Eq. (12) will not have a nonzero solution unless the matrix $D$ is singular and in general the matrix D will not be singular. To approximate the solution of Eq. (12), we consider an eigenvalue problem:

$$
\text { Dy }=\lambda y
$$

We will consider the solution of Eq. (14) that corresponds to the eigenvalue with the smallest magnitude to be the best approximate solution to Eq. (12). Suppose that $y$ is the eigenvector that corresponds to the eigenvalue with the smallest magnitude. Define $\rho$ by:

$$
\rho^{2}=\sum_{i=2}^{n+1}\left(y_{i}\right)^{2}
$$

Then:

$$
\begin{gathered}
d=y_{1} / \rho \\
a_{i}=y_{i+1} / \rho, \text { for } i=1 \text { to } n
\end{gathered}
$$




\section{NUMERICAL EXPERIMENTS}

We have developed a method for obtaining a least squares fit of a hyperplane to uncertain data. In this section, we will apply our method to synthetic data sets in three dimensions. We will compare the results for our eigenvalue method with a partial information approach (neglecting one equation at a time). The partial information approach does not produce a single solution (it produces four different solutions for the unknown parameters). We will find that the parameters determined by our eigenvalue method are within the range of the four sets of parameters determined by the partial information approach.

We consider a plane in three dimensional space with parameters: $a=(0.5774$, $0.5774,0.5774$ ) and $d=1.0$. To create a synthetic data set, we calculate 81 data points with random errors that are proportional to a parameter $(\sigma)$. Given the data set and the weights $\left(w_{j}=1 / 81\right)$, we can calculate the $B$ vector and the $A$ matrix and solve the eigenvalue problem. The parameters a and $d$ are displayed in Table 1 for five values of $\sigma$. As $\sigma$ increases, two of the parameters $\left(a_{1}\right.$ and $\left.a_{2}\right)$ decrease and two increase ( $a_{3}$ and $\left.d\right)$. The parameter values have small variations as the error parameter $(\sigma)$ increases.

Table 1. The parameters a and $\mathrm{d}$ for five synthetic data sets.

\begin{tabular}{|c|c|c|c|c|}
\hline$\sigma$ & $\mathrm{a}_{1}$ & $\mathrm{a}_{2}$ & $\mathrm{a}_{3}$ & $\mathrm{~d}$ \\
\hline 0.0 & 0.5774 & 0.5774 & 0.5774 & 1.0000 \\
\hline 0.1 & 0.5768 & 0.5755 & 0.5798 & 1.0535 \\
\hline 0.2 & 0.5763 & 0.5741 & 0.5816 & 1.1075 \\
\hline 0.4 & 0.5755 & 0.5723 & 0.5842 & 1.2180 \\
\hline 0.8 & 0.5733 & 0.5701 & 0.5885 & 1.4529 \\
\hline
\end{tabular}

For a problem in three dimensional space, the matrix $D$ is 4 by 4 . We can obtain four solutions to the over determined problem by neglecting one row at a time. When the error parameter is zero, the matrix $\mathrm{D}$ is singular and both approaches give the same result.

When the error parameter is positive, the matrix $D$ is not singular and the two approaches give different results. We compare our eigenvalue method with the approach of neglecting one equation at a time in Figures 1 and 2. Figure 1 displays the values of the parameter $a_{1}$ calculated using the two approaches (the eigenvalue method results are denoted by a plus symbol while the partial information results have a square symbol) for several values of the error parameter. Figure 2 displays the values of the parameter $d$ 
calculated using the two approaches for several values of the error parameter. The partial information approach produces four sets of results that have considerable dispersion. Our eigenvalue method automatically produces a single result that lies within the range of the four sets of parameters determined by the partial information approach. The partial information approach neglects information and determines four different planes. Our eigenvalue method does not neglect any information and automatically produces a single result that is an "average" of the four planes determined by the partial information approach.



Fig. 1. The values of the parameter $a_{1}$ calculated using the eigenvalue method (plus symbol) and the partial information approach (square symbol) for several values of the error parameter. 




Fig. 2. The values of the parameter $d$ calculated using the eigenvalue method (plus symbol) and the partial information approach (square symbol) for several values of the error parameter. 


\section{CONCLUSIONS}

We have developed a method for obtaining a least squares fit of a hyperplane to uncertain data. The method uses standard techniques (eigenvalues and eigenvectors) to estimate the parameters for the hyperplane. We have estimated parameters for five sets of synthetic data. The parameter values have small variations as the error parameter increases. Our method appears to be robust (capable of producing good estimates of parameters for noisy data sets). 


\section{ACKNOWLEDGMENT}

Research sponsored by the Engineering Research Program, Office of Basic Energy Sciences, of the U.S. Department of Energy, under contract DE-AC05-84OR21400 with Martin Marietta Energy Systems, Inc. and by the Naval Air Warfare Center Aircraft Division/API Group, Technology and Development Office, under Interagency Agreement No. 2072-E123-A1 with the U.S. Department of Energy. 


\section{INTERNAL DISTRIBUTION}

1. J. E.Baker

2. M. Beckerman

3. C. W. Glover

4. W. C. Grimmell

5. J. P. Jones

6. H. E. Knee

7-11. R. C. Mann

12-16. M. D.Morris

17. E. M. Oblow

18-22. C. E. Oliver

23. L. E. Parker

24-28. F. G. Pin

29. N. S. V. Rao
30-34. D. B. Reister

35. S. Shekhar

36-40. R. F. Sincovec

41. E. C. Uberbacher

42. M. A. Unseren

43-47. R. C. Ward

48. EPMD Reports Office

49-50. Laboratory Records Department

51. Laboratory Records, ORNL-RC

52. Document Reference Section

53. Central Research Library

54. ORNL Patent Office

\section{EXTERNAL DISTRIBUTION}

55. Dr. Peter Allen, Department of Computer Science, 450 Computer Science, Columbia University, New York, NY 10027

56. Dr. John Baillieul, Aerospace and Mechanical Engineering Department, Boston University, 110 Cummington St., Boston, MA 02215

57. Dr. Wayne Book, Department of Mechanical Engineering, J. S. Coon Building, Room 306, Georgia Institute of Technology, Atlanta, GA 30332

58. Professor Roger W. Brockett, Harvard University, Pierce Hall, 29 Oxford St., Cambridge, MA 02138

59. Dr. Steven Dubowsky, Department of Mechanical Engineering, Massachusetts Institute of Technology, Building 3, Room 469A, 77 Massachusetts Ave., Cambridge, MA 02139

60. Professor Donald J. Dudziak, Dept. of Nuclear Engineering, 110B Burlington Engineering Labs, North Carolina State University, Raleigh, NC 27695-7909

61. Mr. Steve Holland, Robotics, B/MD-63, General Motors Corporation, NAO Manufacturing Center, 30300 Mound Rd., Warren, MI 48090-9040

62. Dr. Avi Kak, Department of Electrical Engineering, Purdue University, Northwestern Ave., Engineering Mall, Lafayette, IN 47907

63. Professor Takeo Kanade, Computer Science and Robotics, Carnegie Mellon University, Pittsburgh, PA 15213-3890

64. Dr. James E. Leiss, Route 2, Box 142C, Broadway, VA 22815-9303

65. Dr. Oscar P. Manley, Division of Engineering, Mathematical, and Geosciences, Office of Basic Energy Sciences, ER-15, U.S. Department of Energy Germantown, Washington, DC 20545

66. Professor Neville Moray, Department of Mechanical and Industrial Engineering, University of Illinois, 1206 West Green St., Urbana, IL 61801

67. Mr. Peter M. O'Donnell, Naval Air Engineering Center, Code 02T (POD), Lakehurst, NJ 08733-5000

68. Mr. Steve Singer, NAWAIRWARCENACDIVLKE, Code 02T (SS), Bldg. 562, Room 310, Lakehurst, NJ 08733-5000

69. Dr. Wes Snyder, Department of Radiology, Bowman Gray School of Medicine, N.C. Baptist Hospital School of Medicine, 300 S. Hawthorne Dr., Winston-Salem, NC 27103 
70. Professor Mary F. Wheeler, Department of Mathematical Sciences, Rice University, P.O. Box 1892, Houston, TX 77251

71. Office of Assistant Manager for Energy Research and Development, U.S. Department of Energy, Oak Ridge Operations Office, P.O. Box 2001, Oak Ridge, TN 37831-8600

72-73. Office of Scientific and Technical Information, P.O. Box 62, Oak Ridge, TN 37831 




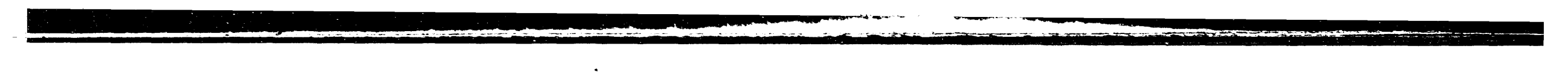

